\title{
Nasal Inhaler Dosage Form
}

National Cancer Institute

\section{Source}

National Cancer Institute. Nasal Inhaler Dosage Form. NCI Thesaurus. Code C91154.

A medication delivered as a mist to be inhaled through the nose as a measured dose 